\title{
Europas Identität (gestern) - Europas Krise (heute): ein Themenwechsel als Indiz für einen Paradigmenwandel?
}

\author{
Heinrich Schneider*
}

\section{Europäische Identität: Aufstieg und Niedergang eines Diskurses}

Alles hat seine Zeit, so das biblische Buch Kohelet. Das gilt auch für europapolitische Diskursgegenstände, besonders für das Thema europäische Identität. Als ihm gegen Ende 1973 die Außenminister der damaligen Europäischen Gemeinschaften ein offizielles Dokument widmeten, ${ }^{1}$ löste das kaum politische Debatten aus, schon gar nicht wissenschaftliche. ${ }^{2}$ Die gab es erst Jahrzehnte später. Inzwischen ist die Konjunktur verebbt.

Den Boom löste vor 20 Jahren der Entschluss der Politik aus, die Gemeinschaften, die bislang der Wirtschaftsintegration dienten, und die Regime intergouvernementaler Zusammenarbeit enger zu verklammern und die Einigungspolitik auf eine neue Stufe zu heben. Von einer ,Politischen Union“ war die Rede. Sie würde stärkerer Bindekräfte bedürfen. Woher sollten sie kommen? Waren neue Motivkräfte der Integrationsvertiefung aktivierbar, die nicht auf elitäre Zirkel von Politikern und Experten beschränkt blieben? Auf welche ideellen Ressourcen und auf welche handfesten Interessen könnte sich dieses Unterfangen stützen?

Die Vollendung des Binnenmarktes würde, meinte man, eine Dynamik freisetzen, die das Integrationssystem sprengen mochte, falls den Zentrifugalkräften nicht genuin europäische Zentripetalkräfte entgegengestellt werden. Im damaligen Jargon gesprochen: Die negative Integration (die deregulierende Entfesselung der Marktkräfte) führt tendenziell zur Erosion des Gemeinsamen Marktes, wenn die Desintegrationsdynamik nicht eingedämmt, womöglich in eine positive Integrationsdynamik umgewandelt wird, durch eine Wirtschafts- und Währungsunion (WWU). Beim ersten Anlauf zu einer solchen, auf der Basis des WernerPlans (1970), war zweierlei unstrittig: Erstens, dass die WWU eine kohärente, gemeinsame Wirtschafts- und Finanzpolitik einzuschließen hätte. Zweitens jedoch, dass eine solche gegen das Widerstreben wichtiger Kräfte konzipiert und durchgesetzt werden müsste - angesichts erheblicher Diskrepanzen der nationalen und regionalen Wirtschaftsstruktur, der unterschiedlichen mitgliedstaatlichen und branchenspezifischen Interessenlagen sowie der gegensätzlichen ordnungspolitischen Positionen. Das vorgesehene ,wirtschaftspolitische Entscheidungsträgergremium“ müsste ,unabhängig und im gemeinschaftlichen Interesse“

* Prof. Dr. Heinrich Schneider, Universität Wien; Institut für Europäische Politik, Berlin.

1 Dokument über die europäische Identität, angenommen von den Außenministern der Mitgliedstaaten der Europäischen Gemeinschaft am 14. Dezember 1973 in Kopenhagen, abgedruckt unter anderem in: Europa-Archiv 2/1974, S. D 50-D 53. Zufolge der „Verlautbarung der Konferenz der Staats- und Regierungschefs“ wollte man damit vor allem den gemeinsamen Willen bekräftigen, „dass Europa in den wichtigen Angelegenheiten der Welt mit einer Stimme spricht“. Vgl. Verlautbarung der Konferenz der Staats- und Regierungschefs der Mitgliedstaaten der Europäischen Gemeinschaften in Kopenhagen am 14. und 15. Dezember 1973, abgedruckt in: Europa-Archiv 2/1974, S. D 54-D 56, hier S. D 54.

2 Ernsthafte Würdigungen unternehmen zum Beispiel Armin von Bogdandy: Europäische und nationale Identität: Integration durch Verfassungsrecht, in: Veröffentlichungen der Vereinigung der Deutschen Staatsrechtslehrer, Band 62, Berlin 2003, S. 156-193, hier S. 177ff. und Wolfgang Schmale: Geschichte und Zukunft der Europäischen Identität, Stuttgart 2008. 
neue Gemeinschaftsbefugnisse erhalten. ${ }^{3}$ Kraft seiner Machtausstattung sollte es das Widerstreben effektiv bändigen können, wozu es eine eigenständige Legitimitätsbasis erhalten müsste.

Als rund zwei Jahrzehnte später die WWU erneut auf die Tagesordnung kam, sah man das Problem deutlicher. Wohlmeinende Autoren meinten, es wäre am besten, ein starker transnationaler oder übernationaler Gemeinsinn könnte dieses Widerstreben von vornherein eindämmen oder überbieten und so die Legitimität der gemeinsamen Wirtschaftspolitik tragen. Dazu schien eine belastbare europäische Identität erforderlich, ein kollektives politisches Selbstbewusstsein europäischer Bürger, sodass in deren Namen ${ }^{4}$ effektiv ,policies ‘ vorangetrieben werden können, nach Maßgabe eines übergeordneten Gemeinwohls und unter Inanspruchnahme transnationaler Solidarität. Ohne eine solche Identität würde sich das politische System einer WWU nicht halten können. Das regte zum Weiterdenken an: Die Integrationsvertiefung erforderte einen Bedarf an europäischem Wir-Bewusstsein. Unter welchen Umständen könnte es artikuliert oder wachgerufen werden? Auf welche kulturhistorischen, sozialethischen oder politisch-ideologischen Rechtfertigungen konnte man sich berufen, aber auch auf welche handfesteren Interessen?

Das Anliegen ließ sich als europäisches Analogon zur vorangegangenen Herausbildung des Nationalbewusstseins begreifen. Freilich scheute man sich, die entsprechende Begrifflichkeit schlichtweg auf die europäische Ebene zu heben - dies hätte ja die Postulierung einer einzigen europäischen Nation bedeutet, also die Infragestellung der überkommenen nationalen Identitäten. Aus guten Gründen gab es dagegen massive Bedenken.

So kam es zu einer weniger provozierend wirkenden, weil abstrakter klingenden Behandlung der Sache unter Zuhilfenahme des Identitätsbegriffs. Weil Funktion und Stellenwert dieses Begriffs in diesem Kontext ohne Weiteres einsichtig waren, ${ }^{5}$ schoben viele Diskurspartner Bedenken gegen die Verwendung des „Plastikworts Identität“ beiseite. ${ }^{6}$ Oft machte man sich die Sache leicht, indem man sich auf Komplexitäten und Untiefen des Begriffs und der Sache, auf die in grundlegenden sozial- und politiktheoretischen Studien hingewiesen worden war, nicht einließ. ${ }^{7}$

Die Konjunktur des Themas war auch weiteren Antrieben zu verdanken. Es kam zu einer erheblichen Ausweitung der Interdisziplinarität europawissenschaftlicher Auseinandersetzungen. Jahrzehntelang war die Szenerie vor allem vom Nebeneinander der ökonomischen, der juristischen und der politikwissenschaftlichen Debatten geprägt, eher selten meldeten sich auch Historiker und Soziologen zu Wort. Nun aber sahen gerade diese und außerdem auch Sozialpsychologen, Kulturanthropologen, Philosophen und Vertreter weiterer Human-

3 Bericht an Rat und Kommission über die stufenweise Verwirklichung der Wirtschafts- und Währungsunion in der Gemeinschaft „Werner-Bericht“, Luxemburg 8. Oktober 1970, Sonderbeilage zum Bulletin 11-1970 der EG, S. 13-73.

4 Vgl. Alfred Grosser: In wessen Namen? Grundlagen politischen Entscheidens, Tübingen 1969.

5 Vgl. Heinrich Schneider: Europäische Identität: Historische, kulturelle und politische Dimensionen, in: integration 4/1991, S. 160-176; Heinrich Schneider: Nationale und europäische Identität im Widerstreit?, in: Gerhard Eickhorn (Hrsg.): Ungarn und Deutschland im künftigen Europa, Bonn 1991, S. 35-49.

6 Nur ganz wenige Autoren, die sich mit europäischer kollektiver Identität befassten, gönnten dem gehaltvollen 680-Seiten-Opus von Lutz Niethammer eine bibliografische Erwähnung; ernsthaft auseinandergesetzt hat sich mit ihm allem Anschein nach kaum einer von ihnen. Vgl. Lutz Niethammer: Kollektive Identität - Heimliche Quellen einer unheimlichen Konjunktur, Reinbek bei Hamburg 2000. Als „Plastikwort“ bezeichnet Niethammer „Identität“" im Anschluss an Uwe Prörksen: Plastikwörter, 2. Auflage, Stuttgart 1992. Auch Karl Dietrich Bracher sprach sich damals massiv gegen die Verwendung des Begriffs aus.

7 Vgl. zum Beispiel Roland Robertson/Burkart Holzner (Hrsg.): Identity and Authority. Explorations in the Theory of Society, Oxford 1980; aus der geisteswissenschaftlich-hermeneutischen Literatur zum Beispiel Odo Marquard/Karlheinz Stierle (Hrsg.): Identität, Poetik und Hermeneutik, Band 8, 2. Auflage, München 1996. 
und Geisteswissenschaften die Chance, eigene Beiträge in die akademische und politikbezogene Europadebatte einzubringen, was zugleich geeignet war, ihre „Gesellschaftsrelevanz“ öffentlich darzustellen. ${ }^{8}$ So kam es zu einer Flut von Beiträgen sehr verschiedener Art. Das wachsende Interesse an den ideellen und motivationalen Erfordernissen einer europäischen ,polity ' aktivierte in erster Linie Autoren, die in der Integrationsforschung zu Hause waren.

Es kam überdies zur Ausweitung des Blickfeldes über das bisherige Terrain hinaus:

- Aufgrund der Wende (1989/90) war ein neuer Bedarf an inhaltlichen Definitionen des Europäischen aufgetreten. Nun erhielt die Vertragsbestimmung, ,jeder europäische Staat“ könne den Beitritt beantragen, besondere Brisanz: Vor der Wende war nur an die Kandidatur westlicher Staaten zu denken - wer kam darüber hinaus nun infrage? Mit dem Ende des Ost-West-Konflikts verlor zudem die antisowjetische Solidarität ihre transnationale Bindekraft. ${ }^{9}$ Die Integration benötigte sozusagen eine neue Art von kohäsionssicherndem Kitt.

- Die Frage nach der Bedeutung und den Chancen des Fortbestands der kulturellen und der politisch-zivilisatorischen Eigenart Europas angesichts der ,multikulti'-Problematik, nicht zuletzt aufgrund der Immigration aus nicht europäisch (namentlich muslimisch) geprägten Kulturräumen. ${ }^{10}$

- Die Kontroverse um die Relevanz religiöser Erbschaften gab der Frage nach einer europäischen Identitätssubstanz zusätzliche Bedeutung, als im Verfassungskonvent über die ausdrückliche Bezugnahme auf dieses Erbe oder gar über eine „nominatio Dei“ in der Präambel gestritten wurde. ${ }^{11}$

Immer wieder bezog die Identitätsdiskussion weitere Schlüsselbegriffe ein, politik- und kulturwissenschaftliche: Demokratie, Nation, Staat, aber auch Wertegemeinschaft.

Mittlerweile hat das Thema, wie es scheint, an Interesse verloren; vor allem wohl, weil die Konstituierung einer ,polity “ gescheitert schien. In den Vertrag von Lissabon gingen zwar wesentliche Inhalte des Vertrags über eine Verfassung für Europa (Verfassungsvertrag) ein; hingegen fielen solche Bestimmungen, die die Identität des Gebildes und die Staatsähnlichkeit seiner Grundordnung betont hatten, weg. Die Reform bekräftigte die Dominanz der Mitgliedstaaten im europapolitischen Prozess. Der Staatenverbund wurde abermals festgeschrieben und damit die bestimmende Rolle der nationalen politischen Identitäten für die Politik der Union und für ihre Legitimität.

Schließlich nahm im Zuge der massiven Herausforderung der Europapolitik durch die finanz- und wirtschaftspolitischen Turbulenzen das Krisenmanagement die Aufmerksamkeit und die politische Handlungspotenz der europapolitischen Akteure so sehr in Anspruch, dass die europäische Identität zum Luxusthema wurde - ,primum vivere, deinde philosophari`: Zwar gibt es noch immer einige Stimmen, die sich der Verweisung der Sache ins Ab-

8 Das schien angesichts eines Reputationsverlusts von Wissenschaften, die nicht auf unmittelbar politisch, ökonomisch oder technisch umsetzbare Erkenntnisse ausgehen, wissenschaftspolitisch, aber auch förderungsstrategisch von Belang.

9 Als schon in den 1960er Jahren der künftige Beitritt der Türkei in Aussicht genommen wurde, gab es kaum irgendwelche Diskussionen, die sich mit der Erregung vergleichen ließen, die es anlässlich des Ringens um die Beitrittsverhandlungen gab. Damals galt die Türkei als ,europäischer Staat ${ }^{\text {‘ }}$ - sonst hätte sie nicht noch im Gründungsjahr des Europarats, diesem beitreten können. In der Ära des Kalten Krieges hatte die NATO-Mitgliedschaft für die Einschätzung der Zusammengehörigkeit mit den anderen Staaten des westlichen Europas wohl eine andere Bedeutung als heute.

10 Vgl. dazu beispielsweise Bassam Tibi: Europa ohne Identität? Die Krise der multikulturellen Gesellschaft, München 1998; Claus Leggewie: Multi Kulti: Spielregeln für die Vielvölkerrepublik, 4. Auflage, Berlin 1993.

11 Vgl. zum Beispiel Heinrich Schneider: Die Europäische Union, Kirchen und Religionen: Rückblicke auf eine Kontroverse, in: integration 1/2008, S. 37-46. 
seits entgegenstellen. ${ }^{12}$ Kommt darin eine trotzige Apperzeptionsverweigerung der aktuellen Realität zum Ausdruck? Handelt es sich bei der europäischen Identität nach dem Scheitern des Verfassungsprojekts um ein abgeschlossenes Kapitel, dann mag es immerhin von der Eule der Minerva überflogen werden, auf dass es der Archivierung überantwortet werden kann, bevor es in Vergessenheit gerät. Diese Einschätzung könnte sich als kurzschlüssig erweisen. Vielleicht ereignet sich einmal mehr eine Sequenz, die im Gang der Integrationsgeschichte mehrmals auftrat: Ideen und Projekte wurden irgendwann erstmals ins Spiel gebracht, dann mangels Konsens der Entscheidungsträger von der Tagesordnung abgesetzt, jedoch nach einiger Zeit abermals auf die Agenda gebracht, weil die „Sachlogik“ (wie Walter Hallstein das nannte) zur erneuten Befassung nötigte. Das WWU-Projekt war ein markanter Fall.

\section{Die jüngste Krise, ihre Bewältigung und das Erfordernis von Solidität und Solidarität}

Diesmal scheinen just jene Herausforderungen die Wiedervorlage des Dossiers nahezulegen, deretwegen es ins Abseits geriet, wenngleich anders artikuliert und in verändertem Kontext.

Ein Stichwort dazu lieferten schon seit mehr als einem Jahr Proponenten einer europäischen Wirtschaftsregierung ${ }^{13}$ Manche von ihnen meinten, die jüngste Krise würde geradewegs zur Thematisierung dieses Projekts nötigen, ${ }^{14}$ dessen Geschichte weit zurück reicht.

Dass ein Binnenmarkt der Stabilisierung durch eine Wirtschafts- und Währungsunion bedarf und dass diese eine Art Wirtschaftsregierung erfordert, entsprach der herrschenden

12 Als Beispiel aus der akademischen Welt vgl. Werner Weidenfeld: Die Bilanz der Europäischen Integration 2010, in: Werner Weidenfeld/Wolfgang Wessels (Hrsg.): Jahrbuch der Europäischen Integration 2010, BadenBaden 2011, S. 13-28, hier vor allem S. 19, 23-25, 27. Als Beispiel aus dem kirchlichen beziehungsweise zivilgesellschaftlichen Bereich vgl. die im Vorfeld der Wahlen zum Europäischen Parlament abgegebene Erklärung des Hauptausschusses des Zentralkomitees der deutschen Katholiken vom 27.03.2009 mit der These, ,,dass für den erfolgreichen Fortgang des europäischen Einigungsprozesses die Frage der europäischen Identität von entscheidender Bedeutung ist“. Sie trage ,erheblich dazu bei, die Akzeptanz der notwendigen Zwänge des Systems und eine gegenseitige Loyalität zwischen den Mitgliedern zu ermöglichen und fortzubilden“. Siehe Zentralkomitee der deutschen Katholiken: Europas Identität. Der Beitrag der christlichen Kultur zu Europas Vielfalt und Einheit, in: Zentralkomitee der deutschen Katholiken (Hrsg.): Berichte und Dokumente 2009, Bonn 2010, S. 221-237, hier S. 226-227. Außerdem erschien immerhin Jahre nach dem Verfassungsdebakel das Buch von Schmale: Geschichte und Zukunft der Europäischen Identität, 2008.

13 Siehe zum Beispiel Thomas Mayer: EU-Wirtschaftsregierung gesucht, in: Der Standard, 04.03.2010; Thomas Mayer/Birgit Baumann: „Wirtschaftsregierung" gegen „Schuldenbremse“, in: Der Standard, 18.05.2010. Joschka Fischer meinte im Februar 2010, es sei „ein hoffnungsvolles Zeichen“, dass sich Angela Merkel „beim jüngsten deutsch-französischen Gipfel [...] zum ersten Mal nicht mehr ablehnend gegenüber der Idee einer europäischen Wirtschaftsregierung gezeigt" habe. Siehe Joschka Fischer: Durchwursteln verboten, in: Süddeutsche Zeitung, 22.02.2010. Zur wissenschaftlichen Beurteilung der jüngsten Auseinandersetzungen vgl. Wolfgang Wessels: Der Europäische Rat als Wirtschaftsregierung. Zur französisch-deutschen Kontroverse, in: Bodo Knoll/Hans Pitlik (Hrsg.): Entwicklung und Perspektiven der Europäischen Union. Festschrift für Professor Dr. Rolf Caesar, Baden-Baden 2009, S. 177-192.

14 Im Sommer 2010 war davon besonders häufig die Rede. Die französische Finanzministerin Christine Lagarde plädierte zum Beispiel für eine „Wirtschaftsregierung, die echte Steuerungsfunktionen übernimmt“, sich ,,nicht nur mit der Banken- und Finanzaufsicht befasst, sondern eine koordinierende und effiziente Exekutive ist" und „die Wettbewerbsunterschiede anzugleichen“ hätte. Siehe Michael Kläsgen: Paris drängt auf Wirtschaftsregierung, in: Süddeutsche Zeitung, 14.06.2010. Der Premier- und Finanzminister Luxemburgs, Jean-Claude Juncker, hatte betont, die Europäische Union umfasse ,das einzige Währungsgebiet, das über keine Regierung mit adäquater Gewalt verfügt“", nachdem abermals vorher Präsident Nicolas Sarkozy sich für die auf der Ebene der Staats- und Regierungschefs promovierte Euro-Gruppe stark gemacht hatte (wogegen die deutsche Kanzlerin alle 27 Mitgliedstaaten in dieses Regime einbezogen wissen wollte). Süddeutsche Zeitung: „Wir werden den Griechen keine Ruhe lassen“, Interview mit Jean-Claude Juncker, 13.02.2010; Süddeutsche Zeitung: Madrid will Europa zu Konjunkturhilfen zwingen, 09.01.2010; Financial Times Deutschland: Merkel stützt Koordination für EU-Wirtschaft, 12.01.2010. 
Lehre. Die schon weiter oben erwähnte Folgerung drängte sich auf: Will eine Wirtschaftsregierung eine in sich kohärente Politik entwickeln, dann wird sie da und dort auf Widerstreben stoßen, auch bei mitgliedstaatlichen Regierungen. Sie muss also imstande sein, ihre Politik gegen dieses Widerstreben durchzusetzen - dazu braucht sie Macht. Aber diese Macht bedarf demokratischer Legitimation und Kontrolle. Also braucht die WWU eine ,Politische Union".

In Hans Tietmeyers Augen, und nicht nur in seinen, sollte die Politik der Wirtschaftsregierung marktwirtschaftlich orientiert sein und der Währungsstabilität dienen. Andere sahen das anders. Schon im Ringen um die Ausgestaltung des Wegs zur Endstufe versuchten die Kontrahenten, die Weichen in die ihnen genehme Richtung zu stellen. Das Projekt scheiterte im Trubel der globalen Währungskrise; schon vorher mutete es recht waghalsig an. Es schien, als hätten die Konstrukteure eine Einsicht vergessen, die kein Geringerer als Ulrich Everling schon 1964 prägnant formuliert hatte: Einheitliche Wirtschaftspolitik erfordert eine politische Einheit. „Diese aber muß wachsen und zu einer Einheit der Völker werden, wenn sie Bestand haben soll." ${ }^{15}$ Eine europäische Wirtschaftsregierung kann nicht funktionieren, wenn sie nicht von einer europäischen Identität Rückendeckung erhält. Davon war allerdings 2010 kaum die Rede.

Man konzentrierte sich im letzten Jahr zunächst auf die akute Gefahr: Ein finanzieller Kollaps Griechenlands und womöglich anderer Staaten könnte die Währungsunion zum Scheitern bringen und die Union selbst gefährden - so hieß es immer wieder. ${ }^{16}$

Über alle Bedenken setzten sich die Staats- und Regierungschefs hinweg: Es gebe keine Alternative. Allerdings wird mit dem Rettungsschirm erst einmal Zeit gekauft, aber die Problematik nicht wirklich bewältigt. So kommt es darauf an, ob die Jahre bis zum Auslaufen des 2010 eingeführten Feuerlöschregimes zur Stabilisierung hinreichend effektiv genutzt werden, wie die Volkswirtschaften das verkraften, vor allem aber ob eine Dauerlösung der Probleme zustande kommen wird und wie sie aussieht.

Im Spätherbst 2010 fand ein Streitpunkt besonders viel Aufmerksamkeit: die Ausgabe von „EuroBonds“. Anscheinend kam im Streit darüber eine Grundkontroverse zum Ausdruck: nämlich über die Balance von Soliditätswettbewerb und Solidarität. Die Verfechter der Solidität erklären, die Kombination von Binnenmarktpolitik und Währungsunion sei von ihren Konstrukteuren bewusst darauf angelegt worden, um den Systemwettbewerb der Mitgliedstaaten innerhalb der Union zu intensivieren. Die Unabhängigkeit der Europäischen Zentralbank (EZB) und ihre Verpflichtung auf die Preisstabilität sollte die Staaten zu einer Anpassung ihrer Wirtschafts- und Finanzpolitik an die Prinzipien der Solidität nötigen und dieser Druck sollte den von der vielberufenen Globalisierung ausgelösten noch überbieten. ${ }^{17}$

15 Ulrich Everling: Die Koordinierung der Wirtschaftspolitik in der Europäischen Wirtschaftsgemeinschaft als Rechtsproblem, Tübingen 1964, S. 36.

16 Die Vorgänge konnten von jedem halbwegs aufmerksamen Zeitungsleser verfolgt werden; auf entsprechende Belege wird daher für die folgenden Passagen verzichtet.

17 Europa sollte sich selbst geradezu einer „Superglobalisierung“ aussetzen, der Unionsvertrag und der Stabilitätsund Wachstumspakt sollten die Hebel dazu bieten. Siehe Rolf Caesar/Wim Kösters: Europäische Wirtschaftsund Währungsunion: Europäische Verfassung versus Maastrichter Vertrag, in: Mathias Jopp/Saskia Matl (Hrsg.): Der Vertrag über eine Verfassung für Europa, Baden-Baden 2005, S. 259-274, unter Hinweis auf Wim Kösters: Systemwettbewerb in der EU - Weg zur Angleichung oder politisches Schlagwort?, in: Walter-Raymond-Stiftung (Hrsg.): Wettbewerb in Europa, Berlin 2003, S. 103-121. Dort wird die "Superglobalisierung“ allerdings nur als möglich bezeichnet. Die Regierungen hätten sich den Verpflichtungen des EU-Vertrags und des Stabilitätspakts weitgehend entzogen und keinerlei Anstalten getroffen, den Bürgern die Konsequenzen des damit eingeführten ,neoklassischen " (nicht etwa keynesianischen) Assignments zu erklären und sie dafür zu gewinnen. Tendenziell habe man sich den Nötigungen des Systemwettbewerbs entzogen, das gelte auch für Deutschland (Dieses Urteil wurde mehrere Jahre vor der Aufweichung des Stabilitätspakts gefällt!). 
Ob alle an der Ausarbeitung des Maastrichter Vertragswerks Beteiligten die Dinge so sahen, kann man bezweifeln. Es gab auch ganz andere Konzeptionen. Zum einen wurde bezweifelt, ob das ,System Maastricht“ funktionieren könnte - man müsse mit seinem Scheitern rechnen. ${ }^{18}$ Vielleicht würden Mitgliedstaaten, wenn sie mit Mühe in die Oberliga der Euro-Länder aufgestiegen seien, die Disziplin nicht mehr durchhalten. ${ }^{19}$ Länder, die sich durch den Systemwettbewerb überfordert fühlten, weil sie einer wachsenden Arbeitslosigkeit weder durch Abwertungen noch geld- oder fiskalpolitisch Herr werden könnten, würden die Währungsunion in Gefahr bringen. Zum anderen könne womöglich aus der Not eine Tugend werden. Das war sozusagen die Alternativkalkulation: Den Mitgliedstaaten werde ,,[...] nichts anderes übrigbleiben, als endlich auf der europäischen Ebene eine effektive ,Wirtschaftsregierung' zu schaffen, die [...] auch eine politische Führungsfunktion gegenüber der Geldpolitik beanspruchen müsste, welche die EZB trotz ihrer formellen Unabhängigkeit nicht ungestraft ignorieren dürfte. Wenn dann [...] einzelne Länder dennoch in ernsthafte wirtschaftliche Krisen geraten sollten, so könnten sie trotz des im Maastrichter Vertrag verankerten Haftungsausschlusses sicherlich auf die politische und finanzielle Solidarität aller anderen Mitgliedstaaten rechnen [...]“. So würden gerade „die von der Währungsunion ausgehenden Risiken und Krisen“ „,die Mitgliedstaaten schließlich zur Verwirklichung einer engeren politischen Union zwingen“". ${ }^{20}$ Das liest sich heute fast wie eine Prophetie, wenn es nicht sogar die Mutmaßung nahe legt, manche Akteure hätten sich die Krise im entsprechenden Sinn zunutze gemacht.

\section{Wird die Sanierungsrechnung ohne den Bürger gemacht?}

Was halten von alledem die Unionsbürgerinnen und -bürger? Ohne hinreichende Einsicht in die Notwendigkeit europäischer Solidarität werden sie kaum bereit sein, der Sanierung etwas abzugewinnen. Die einen (zum Beispiel die Deutschen) werden sich um die Früchte ihrer Solidität und ihrer Anstrengungen betrogen fühlen, wenn sie - als Steuerzahler - im Namen Europas für die Sanierung derer aufkommen sollen, die in ihren Augen an ihrer Malaise selbst schuld sind. Die anderen (zum Beispiel die Griechen) werden sich durch eine von der Europäischen Union diktierte Auferlegung von Lohnkürzungen, Steuererhöhungen, durch den Abbau von Sozialleistungen im Namen Europas gedemütigt und ungerecht behandelt fühlen. Das dürfte sowohl hier wie dort die Einstellung zur Europäischen Union überhaupt und im Besonderen zu ihrer Krisenbewältigungspolitik ins Negative beeinflussen.

Wenn solche unmittelbaren Anmutungen die Einstellung der Bürger gegenüber der Europäischen Union überhaupt und zur derzeitigen Krisenbewältigung bestimmen, wäre das arg - schon deswegen, weil das populistischen Demagogen zugutekäme. Durchblick und Urteilskraft wären gefragt. Man mag sich fragen, wie groß (oder klein) seinerzeit die Chance war, dass jene integrationspolitischen Grundentscheidungen, die die Volkswirtschaften und die Wirtschaftsgesellschaft Europas prioritär dem Wettbewerb überantwortet haben, ver-

18 Die Kontroverse ist dokumentiert in Rudolf Hrbek (Hrsg.): Der Vertrag von Maastricht in der wissenschaftlichen Kontroverse, Baden-Baden 1993, vor allem S. 159-187.

19 Bekanntlich führte gerade diese Sorge zum Abschluss des sogenannten Stabilitäts- und Wachstumspaktes. Dass der Aufstieg in die ,Oberliga ‘ von vornherein durch unkorrekte Angaben erschwindelt werden könnte, hatte man allerdings nicht vorhergesehen.

20 So vor rund zwölf Jahren, als die Euro-Einführung unmittelbar bevorstand, Fritz W. Scharpf: Europa nach dem Euro. Ökonomische Integration ohne politischen Fortschritt, in: Internationale Politik 6/1998, S. 39-44, hier S. $39-40$. 
standen und gebilligt wurden. ${ }^{21}$ Eine Zeit lang konnten die Herren des Geschehens den ,permissive consensus' nutzen; das war einmal. Ob und in welchem Maß heute die so oder so Betroffenen erkannt haben (oder erkennen), was zur Euro- und EU-Krise geführt hat und was man von den Konsequenzen halten kann, die daraus gezogen wurden (und werden), steht bislang dahin.

Dies alles gehört zu den Voraussetzungen und zum Umfeld der Gipfelentscheidungen des Jahres 2010. Diese haben die Union in zwei Dimensionen neu ausgerichtet - der Not gehorchend: „Wir haben den Abgrund gesehen und das hat uns verändert“, ,eine neue europäische Gemeinschaft" sei entstanden - so Österreichs Vizekanzler Josef Pröll. ${ }^{22}$

Vieles ist noch offen, zumal auch noch andere Staaten eigene Beiträge zum Thema angekündigt haben. Jedenfalls nehmen wichtige Regierungen ein deutliches wirtschaftspolitisches ,Zusammenrücken“ in Aussicht. Die Harmonisierung könnte wohl funktionieren, so lang die extremen Krisenlagen den Beteiligten noch vor Augen stehen. Beim Nachlassen des Krisenbewusstseins besteht indessen die Gefahr einer Rückkehr zum mentalen status quo ante - es sei denn, das Wir-Bewusstsein (!) und die Solidarisierungsbereitschaft werden durch besondere Vorkehrungen wach gehalten und gestützt.

Das betrifft zunächst die zentralen europapolitischen Akteure, aber nicht nur sie. Werden die Unionsbürger für die Prinzipien und Prozeduren der vertieften WWU nachhaltig Verständnis aufbringen? Kann ein solches System überhaupt nachhaltig effektiv und effizient funktionieren, wenn es von den Bürgerinnen und Bürgern nicht bejaht, sondern allenfalls zähneknirschend hingenommen wird? Vor allem dann, wenn die künftige Unionspolitik immer noch, oder wieder, Durststrecken bewältigen muss?

Wer diese Fragen nicht verdrängt, wird auch die Bedingungen der Möglichkeit eines die Solidarität stützenden Wir-Bewusstseins wieder bedenken müssen. Solidarität und Identität haben einiges miteinander zu tun. ${ }^{23}$ Der Abgesang zum Thema Identität war verfrüht.

\section{Das Thema gewinnt wieder Aktualität - die Debatte von gestern verdient einen Rück- blick}

Im Maastrichter Unionsvertrag steht: Die Entscheidungen der Union werden „möglichst bürgernah" getroffen. Gemeinhin wird das als Umschreibung des Subsidiaritätsprinzips verstanden. Bürgernähe ist aber auch in einem anderen Sinn ein Erfordernis: Schon vor 35 Jahren betonte der ,Tindemans-Bericht“: Viel sei zu tun, damit die Integration ,,vor den Augen der Öffentlichkeit in einem besseren Licht erscheint“ - Europa muss „,dem Bürger näher gebracht werden.“24 Rund zehn Jahre später wurde die Überlegung fortgeführt: Nötig sei ,,das

$21 \mathrm{Zu}$ den Voraussetzungen, Vollzugsweisen und Konsequenzen dieser Grundentscheidungen vgl. zum Beispiel Richard Münch: Die Konstruktion der europäischen Gesellschaft. Zur Dialektik von transnationaler Integration und nationaler Desintegration, Frankfurt Main/New York 2008, S. 364-372, S. 377. Der Autor spricht von einem „Regime des europäischen konstitutionellen Liberalismus“, das zur Verschärfung des „Wettbewerbs zwischen den Individuen um ihre Positionierung in der Gesellschaft" beitrage. Die Installierung des Wettbewerbs zwischen den füreinander zunehmend offen werdenden Wirtschaftssystemen ist dann nur die Einbeziehung der unionsinternen ,internationalen“ Beziehungen in dieses Regime. Befürworter dieser umfassenden europäischen Ordnungspolitik werden heutzutage vielleicht nicht so sehr eine sozusagen naturrechtsäquivalente Rechtfertigung des liberalen Wettbewerbs vorbringen, sondern auch auf die Notwendigkeit verweisen, dass Europa in die Lage kommen könnte, sich in einem zunehmend schärferen globalen Wettbewerb zu behaupten.

22 Nach Hans-Jürgen Schlamp: Zwang zur Harmonie, in: Der Spiegel, 13.09.2010.

23 Dazu statt vieler zum Beispiel Dieter Freiburghaus: Wohin des Wegs, Europa?, Bern 2000, S. 345-346.

24 Leo Tindemans: Bericht über die Europäische Union vom 29.12.1975, in: Heinrich Schneider/Wolfgang Wessels (Hrsg.): Auf dem Weg zur Europäischen Union? Diskussionsbeiträge zum Tindemans-Bericht, Bonn 1977, S. 239-288, hier S. 275-276. 
Entstehen eines europäischen Bewusstseins“, dazu müsse man sich um die „Stärkung des Bildes und der Identität der Gemeinschaft" bemühen. ${ }^{25}$

Soweit man sich darum bemühte, war das Resultat unzulänglich. Als die erste Reform des Maastrichter Vertragswerks bevorstand, der 1997 geschlossene Vertrag von Amsterdam, beauftragten die Verhandlungspartner zunächst eine Reflexionsgruppe mit der Identifizierung der vordringlichen Probleme und Lösungsmöglichkeiten. Nach dem ersten Durchgang machte der Vorsitzende, Carlos Westendorp, zwei wichtige Herausforderungen namhaft: Im „,inneren Kontext“ müsse es vor allem um die „Versöhnung“ der Union mit ihren Bürgern gehen, die „Entfremdung zwischen den Bürgern und der Union“" müsse überwunden werden. Im „äußeren Kontext“" sei es vordringlich, dass die Union „eine äußere Identität erhält“, kraft derer sie zu einem ,globalen Akteur“ werden könne. ${ }^{26}$

Damals waren die Einstellungen der Bürgerinnen und Bürger gegenüber der Europäischen Union beziehungsweise der Europäischen Gemeinschaften und ihrer Politik erheblich positiver als heute. Inzwischen lässt die sozusagen psychologische Bürgernähe der Europäischen Union bei Weitem mehr zu wünschen übrig. Europa ist zwar nicht mehr, weit weg'von den Lebensproblemen der Europäer, aber die Nähe mutet eher unheimlich und beklemmend an. Während Meinungsanalysen in der Frühzeit da und dort (vor allem in Deutschland) geradezu einen europäischen Enthusiasmus und nach dessen Verschwinden den ominösen ,permissive consensus' diagnostizierten, folgte dann die Tendenz einer ,schleichenden Abwendung von Europa. "27 Nun gibt es eher eine negativ aufgeladene Zuwendung, geprägt von Misstrauen und Abneigung. Ob diese Einstellung gestoppt oder sogar in die positive Gegenrichtung umgelenkt werden kann, ist kaum absehbar. Im Fall ihrer Ausweitung und Vertiefung wäre höchste Besorgnis angebracht. Viel wird davon abhängen, ob die Bemühungen um die Krisenbewältigung und in Richtung auf eine der Solidität und der Solidarität verpflichtete Wirtschaftsunion Erfolg haben werden, und zwar nicht nur derart, dass es zu neuen, verbindlichen Verabredungen kommt, im Zuge von Vertragsänderungen oder innerhalb des bestehenden Vertragssystems. Wichtig wäre, dass die Bürgerinnen und Bürger im Hinblick auf Effektivität und Effizienz der Umsetzung wieder Zuversicht fassen - sodass sie im Blick auf die Europapolitik nicht nur Probleme und Ärgernisse, sondern auch glaubwürdige Lösungen wahrnehmen.

Inzwischen ist so gut wie ausgeschlossen, dass das ,Rettungsschirm'-Arrangement 2013 ersatzlos beendet wird; die Chancen für den neuen Krisenbewältigungsmechanismus (European Stability Mechanism, ESM) stehen eher günstig. Die Sanierungsprogramme erlegen den Empfängerstaaten und ihren Einwohnern jedoch so massive Anstrengungen und Wohlstandseinbußen auf, dass das zu starkem Unmut, womöglich zu politischen Krisen und Unruhen führen kann. ${ }^{28}$ Andererseits werden die Bürger jener Länder, deren Steuermittel für die Beistandsleistungen in Anspruch genommen werden, sich die Frage stellen, ob man ihnen abverlangen kann, für die Leichtfertigkeit oder gar die bewusste Trickserei anderer ge-

25 Europa der Bürger: Bericht des Ad-hoc-Ausschusses für das Europa der Bürger an den Europäischen Rat, herausgegeben vom Auswärtigen Amt, Bonn 1985 (sogenannter Adonnino-Bericht), hier vor allem S. 42.

26 Zwischenbericht des Vorsitzenden der Reflexionsgruppe der Regierungskonferenz 1996 vom 24.08.1995.

27 Siehe dazu die Beiträge zum Thema „Die öffentliche Meinung“ in den Jahrbüchern der Europäischen Integration. Vgl. erstmals Thomas Herz: Die öffentliche Meinung, in: Werner Weidenfeld/Wolfgang Wessels (Hrsg.): Jahrbuch der Europäischen Integration 1980, Bonn 1981, S. 284-290.

28 Unmittelbar vor dem Dezembergipfel 2010 wurde kolportiert, die Regierungen einiger in der Schuldenenge befindlicher Länder würden darauf ausgehen, die Unterstützung auch ohne die Erfüllung der Sanierungsaufgaben zu erhalten. Siehe Claus Hulverscheidt/Cerstin Gammelin: Ich bin pleite - bitte rettet mich mal!, in: Süddeutsche Zeitung, 14.12.2010. Angesichts von Generalstreiks gegen die Sparmaßnahmen klingt das nicht unplausibel, wiewohl so etwas vielleicht auch zum taktischen Geplänkel im Gipfelvorfeld gehören mag. Vgl. zum Beispiel Salzburger Nachrichten: Generalstreik und Krawalle in Griechenland, 16.12.2010. 
rade zu stehen - wie auch immer die Konditionen des Beistandes festgelegt werden. ${ }^{29}$ Die Entfremdung zwischen der Union und ihren Bürgern wird mithin kaum behoben werden, sondern eher wachsen, aufgrund des Eindrucks, dass ,Brüssel ' eine Politik betreibt, die sehr handfeste materielle Interessen massiv beeinträchtigt.

Auf einer anderen Ebene liegt die Frage, ob die anstehende Reform der Union, die im Zug der Krisenbewältigung in Richtung auf eine Wirtschaftsunion geht, von den Bürgerinnen und Bürgern verstanden und gebilligt wird. Dies ist heute wichtiger als in der Zeit des ,permissive consensus'. Tatsächlich wird wohl nur eine stark interessierte, politisch gebildete Minderheit sich ein Bild von den Problemen machen, die schon früher anlässlich der Diskussionen über ein derartiges Projekt angesprochen wurden und nun wieder aktuell werden:

- Wird es sich um eine Government-Struktur von der Art handeln, wie sie schon früher als unabdingbares Erfordernis einer Wirtschafts- und Währungsunion galt - und würde eine solche Struktur funktionieren können, angesichts der Inhomogenität des integrierten Wirtschaftsraums?

- Oder wird die Reform zu einem Arrangement für „Governance ohne Government“30 führen, also zum Verzicht auf eine Institution, die in der Orientierung auf ein unionales Gemeinwohl eine von den Mitgliedstaaten zumindest in etwa unabhängige und in sich schlüssige Wirtschaftspolitik entwerfen und durchsetzen kann? Wird ein solches Arrangement zu einer konsistenten Wirtschaftspolitik führen können oder sozusagen im Geflecht der widerspruchsvollen mitgliedstaatlichen Interessen hängen bleiben? ${ }^{31}$

Derlei Sorgen werden wohl eher in der anspruchsvolleren medialen (und akademischen) Auseinandersetzung zur Sprache kommen. Wenn die Europäerinnen und Europäer so sensibel bleiben, wie sie es wurden, wird diese Auseinandersetzung sich auch auf die allgemeine Bewusstseinslage auswirken - sofern diese nicht demagogischen Politikern und Boulevardmedien anheimfällt.

Wie immer: Europäische Solidarität wird ein aktuelles Thema bleiben. Die Diskussion darüber wird die Frage nach wirksamen Motiven nicht ausblenden können. Das spricht dafür, dass auch die Diskussion über die europäische Identität wieder aufgegriffen wird.

\section{Eine zweite Identitätsdebatte - anders als die erste?}

Die zweite Identitätsdebatte wird allerdings in einem veränderten Kontext stehen: Die Erfahrung der Krise, die Diskussion um ihre Deutung und der Streit um den richtigen Weg zu ihrer Bewältigung verändern den Stellenwert der früher geltend gemachten Argumente. Etwas zugespitzt gesagt: Die unmittelbar wahrgenommene oder die von Sachkennern gewonnene und den Bürgerinnen und Bürgern plausibel gemachte Einsicht, dass wir alle notgedrungen ,in einem Boot sitzen “ und nur gemeinsam im Sturm ein rettendes Ufer erreichen können, wird vermutlich im europapolitischen Bewusstsein wichtiger werden als das Raisonnement über historische Erbschaften.

29 Umfragen zufolge wünschen sich bereits 49 Prozent der Deutschen die DM wieder zurück, nur eine weit kleinere Minderheit urteilt über die Einführung des Euro positiv.

30 Zur besagten Formel vgl. James Rosenau/Ernst Otto Czempiel (Hrsg.): Governance without Government. Order and Change in World Politics, Cambridge 1992.

31 Das wäre, ganz abgesehen von der gemeinsamen Steuerung, etwa durch den Europäischen Rat im Euroländerformat, schon dann der Fall, wenn die beabsichtigte ,Härtung ' des Stabilitätspakt-Regimes nicht so zustande käme, wie es angekündigt wurde: mit Frühwarnvorkehrungen (etwa in Form einer Vorlage der nächstjährigen Etatplanung und der mittelfristigen Finanzvorausschau durch die Regierungen an die Kommission, die freilich die nationale Budgethoheit infrage stellen dürfte) sowie wirksameren Sanktionen, die die Selbstabsolution sündiger Regierungen ausschließen, zugunsten eher automatisch wirksamer Sanktionierung. 
Außerdem wird es auch eine Rolle spielen, ob und wie die Proklamation der nun als unauflöslich bezeichneten Schicksalsgemeinschaft hingenommen und begriffen wird:

- als Betonung einer misslichen Tatsache, die man eigentlich den Europapolitikern verdankt (den Konstrukteuren und Durchsetzern der Maastrichter Währungsunion);

- als Beschwörungsformel, die der Festigung und Rechtfertigung dieser Politik und ihrer nun unvermeidlichen Reform dienen soll;

- oder als Versuch, der Union, ihrer Fortbildung und ihrer gedeihlichen Weiterentwicklung ein ideelles Fundament zu geben, das dazu beiträgt, Turbulenzen wie die derzeitigen zu überstehen.

Welche dieser (und vielleicht auch noch anderer) Sichtweisen wirksam ist oder wird, davon dürfte es abhängen, ob und mit welchem Repertoire von Anmutungen und Argumenten die im Zug der seinerzeitigen Identitätsdiskussion zuweilen geforderte, zuweilen beschriebene und analysierte Identitätspolitik Chancen der Wirksamkeit haben wird. Die Thesen, Kontroversen und die behaupteten Resultate der vorangegangenen Identitätsdebatte werden also nicht ohne Weiteres in einer wenig veränderten Neuauflage abermals vermarktet und konsumiert werden können. Aber das ist zunächst nur eine Hypothese. Zu ihrer Überprüfung ist (oder wäre) es nötig, die Identitätsdiskussion der 1990er und anschließenden Jahre zu vergegenwärtigen.

Das kann an dieser Stelle nur in Form eines verallgemeinernden, auf tiefer gehende Einzelwürdigungen verzichtenden Überblicks geschehen, der vor allem dazu dienen könnte, weiße Flecken auf der Argumentationslandkarte sichtbar zu machen und auf zusätzlich ins Spiel zu bringende Fragen hinzuweisen.

Zuerst muss man konstatieren: Es gibt keinen klar darstellbaren Diskussionsstand, der sich aus der Vielzahl der Wortmeldungen und Editionen ableiten ließe. Die Autoren definieren Identität (auch europäische) recht unterschiedlich, manchmal überhaupt nicht ausdrücklich. Oft betrachten sie einander nicht als Diskurspartner, gehen nicht aufeinander ein. Selten werden Positionen, die den eigenen Thesen widersprechen, kritisch aufgegriffen, mit dem Ziel, sie zu widerlegen, als vergleichsweise schwächer zu erweisen oder gar von ihren Autoren zu lernen. ${ }^{32}$ Zwar können Begriffsdefinitionen kontextabhängig gewählt werden, gemäß dem jeweiligen Verwendungszusammenhang und dem Erkenntnisziel. Da das auslösende Moment und der konstitutive Problemzusammenhang des Diskurses ziemlich klar sind, ist der sich zuweilen aufdrängende Eindruck, es werde aneinander vorbei geredet, bedauerlich. Doch gibt es auch sorgsame Begriffsbestimmungen kollektiver Identität. ${ }^{33}$

Im Anschluss an die Begriffsbestimmung liegt eine dimensionale Analyse der Sache nahe. Üblich ist zum Beispiel einerseits die Unterscheidung kultureller und politischer Identität. ${ }^{34}$ Die letztere wird unterschiedlich konzipiert. Recht markant ist ihre Bestimmung als

32 Das gilt oft auch für die in einem einzigen Sammelband enthaltenen Beiträge, wiewohl man manchmal den Eindruck hat, die Herausgeber seien nicht auf ein Sammelsurium ausgegangen, sondern hätten eine klare Perspektive zugrunde gelegt.

33 Vgl. neben dem bereits erwähnten ,klassischen“ Band von Robertson/Holzner: Identity and Authority, 1980, insbesondere die Analysen von Armin von Bogdandy. Er unterscheidet zwei manchmal durcheinander gebrachte Bedeutungszweige und verweist auch auf den häufigen „kryptonormativen“ Gebrauch des Ausdrucks. Andere Autoren insistieren hingegen darauf, dass die Seins- und die Sollensdimension europäischer Identität zwei Seiten einer Medaille seien. So zum Beispiel Werner Weidenfeld. Vgl. Bogdandy: Europäische und nationale Identität, 2003, hier vor allem S. 160-162; Werner Weidenfeld: Reden über Europa - die Neubegründung des europäischen Integrationsprojekts, in: Julian Nida-Rümelin/Werner Weidenfeld (Hrsg.): Europäische Identität: Voraussetzungen und Strategien, Baden-Baden 2007, S. 11-28.

34 Sie wird mehrmals von Thomas Meyer betont, der massiv dafür plädiert, die „Identität Europas“ nur als eine politische zu verstehen - die Suche nach einer kulturellen Identität Europas sei abwegig. Andere Autoren meinen hingegen, die Substanz auch der politischen Identität Europas beruhe auf der kulturellen. Vgl. Thomas Meyer: Die Identität Europas. Der EU eine Seele?, Frankfurt Main 2004. 
Bewusstsein der unausweichlichen Schicksalsgemeinschaft und der ,gleichen Betroffenheit". ${ }^{35}$ In eine andere Richtung geht die Gegenüberstellung von ontologisch-essentialistischen und konstruktivistischen Verständnisweisen kollektiver Identität. Manche Autoren sprechen der europäischen Identität einen der subjektiven Auseinandersetzung vorgegebenen Seinsstatus zu: Sie wird nicht entworfen, sondern erkannt. ${ }^{36}$ Dem gegenüber begreifen andere Autoren kollektive Identitätskonzepte (die europäische Identität eingeschlossen) als Konstrukte, die zwar das Denken, Fühlen, Wollen und Handeln von Menschen beeinflussen können, was aber nicht zu einer ,verdinglichenden Hypostasierung der Identität“ führen dürfe. ${ }^{37}$

Dieser Gefahr entgehen Sichtweisen, die auf die historische Kontextabhängigkeit und auf die Notwendigkeit verweisen, dass der Gehalt des Konstrukts immer wieder neu erarbeitet (erstritten oder ausgehandelt) werden muss, sodass europäische Identität eher Prozesscharakter hat und nicht als eine der Umdeutung enthobene Orientierungsgröße begriffen werden kann, die man sozusagen nur gewahren und erwerben muss, um sie zu besitzen.

Eher zum Eingemachten führt die Frage, ob es denn überhaupt einen Bedarf an europäischer Identität und an ihrer Stärkung - an ihrer Einbringung in die politische ,Sinnwelt“38 der Europäer - gibt. Viele Diskussionsteilnehmer bejahen das als selbstverständlich. Dann bietet es sich an, den Möglichkeiten einer aktiven Förderung des entsprechenden Bewusstseins nachzugehen, also Strategien, vielleicht Rezepte, der Identitätspolitik zu erarbeiten. ${ }^{39}$ Das wird vor allem dann so gesehen und gehandhabt, wenn man erstens kollektive Identität für ein Erfordernis eines jeden Gemeinwesens hält, und wenn man die Europäische Union als bereits existierendes oder als werdendes Gemeinwesen betrachtet.

So sehen das indessen keineswegs alle Diskussionsteilnehmer. Schon über den Bedarf des überkommenen Staates an kollektiver Identität (zumeist artikuliert in einem die Zusammengehörigkeit bekundenden Erinnerungsschatz und in der Bejahung einer Wertegemeinschaft) wird kontrovers diskutiert. Ein Kenner der Thematik bezeichnet die kollektive Identität als „das elementare Konstruktionsprinzip moderner Gesellschaften“.40 Ein anderer,

35 Sie wird mehrmals von Thomas Meyer betont, siehe zum Beispiel: Meyer: Die Identität Europas, 2004. (Meyer hat diese These auch in anderen Veröffentlichungen vertreten). Sie drückt sich erstens im Bewusstsein der Zugehörigkeit zu der (real bestehenden oder unterstellten) Schicksalsgemeinschaft aus und zweitens im Einvernehmen über ein „gemeinsames Projekt“. Politik ist, mit Arnold Toynbee zu reden, „response“ auf „,challenges“, die ein Kollektiv wahrnimmt. Siehe Arnold Tonybee: Der Gang der Weltgeschichte, Stuttgart 1958.

36 Werner Weidenfeld spricht von der ,geschichtlich geformten Physis europäischer Identität“, die als ,Wesensmosaik" begriffen werden müsse. Nimmt man die Wortwahl (,physis“) ernst, dann verweist sie auf die klassische Gegenüberstellung von „physis“ (objektiver Wesensnatur) und „,nómos“ oder „,thésis“ (Setzung). Der europäischen Identität wird damit ein Seinsstatus zuerkannt, wenn gleich sie auch „Metamorphosen“ erfahren hat. Vgl. Weidenfeld: Reden über Europa, 2007.

37 Das Zitat bei Peter L. Berger/Thomas Luckmann: Die gesellschaftliche Konstruktion der Wirklichkeit, Frankfurt Main 1969, S. 185. Die Konsequenz daraus lautet: „Eine europäische Identität existiert, wenn sie als solche von Individuen und Gruppen [...] gedacht und konstruiert wird“. Menschen werden, wie schon Epiktet feststellte, nicht von den Dingen bewegt, sondern von Meinungen über die Dinge. Für unseren Gegenstand heißt das: „Eine europäische Identität existiert, wenn sie als solche von Individuen und Gruppen [...] gedacht und konstruiert wird." Vgl. Orietta Angelucci von Bogdandy: Europäische Identitätsbildung aus sozialpsychologischer Sicht, in: Ralf Elm (Hrsg.): Europäische Identität: Paradigmen und Methodenfragen, Baden-Baden 2002, S. 111-134.

38 Zur Bedeutung des Begriffs siehe Berger/Luckmann: Die gesellschaftliche Konstruktion der Wirklichkeit, 1969.

39 Julian Nida-Rümelin und Werner Weidenfeld erklären zum Beispiel im Vorwort ihres Sammelbandes: Die Notwendigkeit einer europäischen Identität (sowie ihrer Stärkung) sei Voraussetzung und Ausgangspunkt. Vgl. Julian Nida-Rümelin/Werner Weidenfeld: Vorwort, in: Julian Nida-Rümelin/Werner Weidenfeld (Hrsg.): Europäische Identität: Voraussetzungen und Strategien, Baden-Baden 2007, S. 7-8.

40 So Weidenfeld: Reden über Europa, 2007; und in einer Reihe weiterer Veröffentlichungen. 
nicht weniger ausgewiesener, hält staatliche „Identitätszumutungen“ für problematisch, sie fordern womöglich ,die Seele des Bürgers“; der Staat darf um die Identifikation des Bürgers mit seinen Grundwerten ,werben“, er darf diese aber nicht verlangen, wohl freilich Loyalität als Rechtsgehorsam. ${ }^{41}$ Gegenüber dem Leitbild des Bürgers, das der Hegelschen Theorie des substanziell sittlichen Staates entspricht, ${ }^{42}$ zieht er Kants Konzeption des Gemeinwesens vor, das am langfristigen Eigeninteresse der Bürger ausgerichtet ist. ${ }^{43}$ Es wäre übrigens ein Irrtum solche Auffassungen nur explizit liberalen Denkern zuzurechnen. ${ }^{44}$

Dass eine politische Identitätsschwäche sogar Vorteile mit sich bringen kann, wird auch von anderen Autoren vermerkt: „Ein solches Gemeinwesen ist gezwungen, seine Existenz und seine Politik ständig neu vor grundsätzlich eigeninteressierten Bürgerinnen und Bürgern zu rechtfertigen “45 - es könnte freilich zynisch klingen, wenn man dies mitten in der derzeitigen Akzeptanzkrise der Europäischen Union zitiert. Womöglich lässt sich das Argument umkehren: Der Politik einer etablierten und eher unangefochtenen ,polity ' mag die Nötigung, sich immer wieder um die Anerkennung und um das Vertrauen zu bemühen, gut tun. Für angefochtene politische Entitäten wie zum Beispiel die Weimarer Republik oder das Zerreißproben zwischen Flamen und Wallonen ausgesetzte Belgien ist der Legitimationsund Akzeptanzbedarf größer - und auch für die Europäische Union. So könnte sie mehr Identifikationsbedarf haben als, normalerweise, ein Staat. Wird ein solcher Bedarf bejaht, dann stellen sich mehrere weitere Fragen:

Erstens: Ist dieser Bedarf bereits einigermaßen gedeckt oder unterentwickelt? Die Antwort hängt nicht zuletzt davon ab, wie man den status quo der Europäischen Union begreift und bewertet: Ist der real existierende Staatenverbund auf Dauer stabilisierbar oder bedarf er der Umgestaltung - etwa gemäß der Forderung „Mehr Europa!“ oder in Richtung auf eine Rückbildung der derzeitigen Integrationsdichte und der supranationalen Elemente? Ein groBer Teil der Plädoyers zugunsten des (Mehr-)Bedarfs geht davon aus, dass die Europäische Union bereits eine , polity ' ist oder im Begriffe steht, eine solche zu werden, oder aber zu einer solchen umgeformt werden sollte. Besonders beliebt ist das Argument: Nur mit einer europäischen Identität ,im Rücken“ kann die Union die für eine erfolgreiche Politik unerlässli-

41 Bogdandy: Europäische und nationale Identität, 2003.

42 In Hegels ,politischem Staat“ hat der Bürger das Bewusstsein, dass sein substanzielles und besonderes Interesse im Interesse und Zweck des Staates bewahrt und enthalten ist, sodass Besonderes und Allgemeines eine Einheit bilden; unterschieden vom „bloßen Not- und Verstandesstaat“, wie er der bürgerlichen Gesellschaft entspricht, und dessen philosophische Theorie er Kant zuschreibt. Siehe vor allem Hegels Rechtsphilosophie.

43 Bogdandy verweist auf Kants These (,Zum ewigen Frieden“), dass eine Staatsordnung auch ,für ein Volk von Teufeln“" geeignet sein muss. Den Bürgern eine Zivilreligion, etwa in Form der Identifizierung mit den konstitutionellen Grundwerten zuzumuten, wäre verfehlt; Bogdandy zitiert die 1. Kammer des Ersten Senats des Bundesverfassungsgerichts vom 24.03.2001: „Die Bürger sind rechtlich nicht gehalten, die Wertsetzungen der Verfassung persönlich zu teilen. “ Loyalität gegenüber der Rechtsordnung ist Pflicht, ,Wertloyalität“ darf nicht erzwungen werden. Bogdandy schließt sich der Meinung an, eine große Bevölkerungskreise umgreifende „substantielle Homogenität“ könne sich für Minderheiten freiheitsgefährdend auswirken. Vgl. Bogdandy: Europäische und nationale Identität, 2003; BVerfG: 1 BvQ 13/01 vom 24.03.2001, Rn. 24.

44 Vgl. die Thesen des konservativen katholischen Philosophen Robert Spaemann über die „Gefährlichkeit der Rede von der Wertegemeinschaft“. Ein Staat, der sich als ein solcher versteht, verhält sich „,als Großkirche, die Dissidentengemeinschaften ausschließt“. Siehe Robert Spaemann: Europa - Wertegemeinschaft oder Rechtsordnung?, in: ,Zeit_Schrift“ 9/2001; „Das künftige Europa wird nur dann eine Rechtsgemeinschaft sein können, in der alle Bürger der Länder europäischer Traditionen ein gemeinsames Dach finden - wenn es Gemeinschaften mit gemeinsamen Wertschätzungen ermöglicht und schützt, selbst aber darauf verzichtet, eine Wertegemeinschaft zu sein."

45 Achim Hurrelmann: Die Europäische Union als Verfassungsgemeinschaft? Optionen und Gefahren konstitutioneller Identitätspolitik, in: Julian Nida-Rümelin/Werner Weidenfeld (Hrsg.): Europäische Identität: Voraussetzungen und Strategien, Baden-Baden 2007, S. 211-230, hier S. 224. 
che Handlungsfähigkeit erwerben oder behalten, denn nur dann sind Entscheidungen auch gegen das Widerstreben betroffener über Macht verfügender Akteure akzeptabel und durchsetzbar (also zum Beispiel Mehrheitsentscheidungen im Rat). ${ }^{46}$

Zweitens: Kann man die europäische Identität nach dem Vorbild der nationalen verstehen oder konstruieren oder ist das abwegig? Nur in Grenzen gibt es im Diskurs darüber eine so starke Betonung der Differenz wie in der juristischen , sui generis'-Debatte und angesichts des seit vielen Jahren wiederholten Postulats, die Integrationsdiskussion müsse sich endlich von der Orientierung am überkommenen Staat (als analytisch verwendetem Modell oder als bundesstaatliches Zielbild) lösen. ${ }^{47}$

Drittens: Wie verhält sich eine Stärkung der europäischen Identität zur orientierenden und motivierenden Kraft anderer kollektiver Identitäten? In manchen Epochen des ,polity building' ging man davon aus, dass es nur eine Bezugsgröße der politischen Loyalität geben könne. Dies war das identitätstheoretische (oder politisch-psychologische) Korrelat zu der Lehre, in einem mehrstufigen (föderalen) Gemeinwesen könne die Staatsgewalt nur dem Ganzen oder den Teilen (dem Bund oder den Gliedern) zukommen. Es handle sich um ein Nullsummenspiel. Derlei Vorstellungen werden heute nur noch selten vertreten. ${ }^{48}$ Häufig wird betont, dass Menschen allemal (und insbesondere in neueren Zeiten) unterschiedlichen Gemeinschaften angehören, woraus sich jeweils, commitments ‘ ergeben. Das gelte auch für die politische Sphäre: Schon das nationale Gemeinwesen ist in gewisser Weise ein Mehrebenensystem, mit mindestens einer kommunalen und einer nationalen Ebene, in föderalen oder bundesstaatsähnlichen Systemen (wie Spanien mit seinen autonomen Communidades) kommt eine intermediäre Ebene hinzu. Wohlmeinende Autoren halten ein Miteinander der Identifikationen für möglich, eine gegenseitige Stützung und Stärkung für wünschenswert: Die Frage, was im Konfliktfall geschieht und wie ein solcher konstruktiv so bewältigt werden kann, dass das Ende nicht die Liquidation (oder allenfalls die schonungsvolle Emeritierung) einer der Ebenen bringt, wird kaum gestellt. Echte Föderalisten betrachten die nationale Identität als Konstrukt zur Rechtfertigung eines partikularen Kollektivegoismus, der eigentlich hinter dem supranationalen Gemeinwohl zurückstehen sollte. Advokaten der Konföderation oder des Staatenverbundes, so wie er in ,Karlsruhe ' begriffen wird, sprechen der mitgliedstaatlichen, nationaldemokratischen Identität den Vorrang zu.

Viertens: Was kann man tun, um einen (Mehr-)Bedarf an europäischer Identifikationsbereitschaft zu decken? Antworten auf diese Frage werden in unterschiedliche Richtungen gegeben. Manche geben der Identitätspolitik die Aufgabe, auf die kognitive Dimension der

46 Das impliziert also Skepsis gegenüber einer „Legitimation durch Verfahren“ und gegenüber der normativen Kraft von Vertrags- oder Verfassungsbestimmungen, die Mehrheitsentscheidungen oder überhaupt supranationale Strukturen und Prozeduren vorsehen.

47 Dass die nationalen Identitätskonstruktionen mit den konzeptionellen Angeboten zur europäischen Identität durchaus ähnlich sind kann angesichts der von vornherein gegebenen funktionalen Äquivalenz, wie sie oben im ersten Abschnitt angedeutet wurde, kaum verwundern. Vgl. zum Beispiel Sonja Puntscher Riekmann/Ruth Wodak: „Europe for All“ - diskursive Konstruktion europäischer Identitäten, in: Monika Mokre/Gilbert Weiss/Rainer Bauböck (Hrsg.): Europas Identitäten. Mythen, Konflikte, Konstruktionen, Frankfurt Main/New York 2003, S. 283-303.

48 Immerhin meint Rainer Bauböck, ,mehrfache Mitgliedschaft“ in ,polities“ sei ,unsinnig“, mag man an ,horizontale Überlappung“" oder an ,,vertikale Mehrstufigkeit" denken. Selten wird hingegen auf andere Typen kollektiver Identität zurückgegriffen; man muss da nicht gleich an das Konzert denken, das im Hintergrund der „nationalen Identität“ steht, nämlich des theologischen Begriffs „,corpus mysticum“, das im Zug der Konstruktion des politischen Nationskonzepts einer Immanentisierung und Partikularisierung unterzogen wurde. Vgl. Rainer Bauböck: Im Missverständnis vereint? Asymmetrie in multinationalen Föderationen, in: Monika Mokre/Gilbert Weiss/Rainer Bauböck (Hrsg.): Europas Identitäten. Mythen, Konflikte Konstruktionen, Frankfurt Main/New York 2003, S. 117-143. 
„Sinnwelten“ einzuwirken, im trivialsten Fall durch belehrende Bewusstseinsbildung über identitätsstiftende Gemeinsamkeiten des Erbes, der Betroffenheit von aktuellen und künftigen Herausforderungen, über die einigende Rolle von Visionen, über die Notwendigkeit der Solidarität und Ähnlichem.

Andere setzen weniger auf entsprechende Narrative und Begriffe, sondern - in Anlehnung an die zur Stützung nationaler Identität üblichen Vorkehrungen und Inszenierungen auf die Anmutungskraft von Personifikationen (Präsentation von Amts-, Autoritäts- oder Reputationsträgern, die hochschätzenswertes Europäertum verkörpern), Bildsymbolen, Ritualen, Denkmälern, Gedenktagen - bis hin zu Fahne, Hymne, europäischen Preisen und dergleichen.

Abermals ein weiteres Rezept setzt stärker auf „Doing Europe“ (als auf „Imagining Europe" $),{ }^{49}$ also auf die Anleitung zu aktiver Partizipation in Handlungszusammenhängen, die europäische Gemeinsamkeit bewusst und politisch wirksam werden lässt. So sollten die Bürgerinnen und Bürger „das Bewusstsein vermittelt bekommen, aktive Mitspieler in einem offenen Prozess zu sein“, wozu man ihnen ihre eigene Interessenbetroffenheit klar und die Chance der Beeinflussung dieses Prozesses glaubhaft machen muss. ${ }^{50}$

Es gibt also auch plurifunktionale Instrumente und Prozeduren der Identitätspolitik: Euro-Banknoten und -Münzen verknüpfen ihre Verwender auf handfest materiell bedeutsame Weise, sollen aber zugleich bildhaft anmutenden Symbolgehalt präsentieren; Europawahlen sind Bestandteile des europapolitischen Prozesses und Rituale, die den Wählerinnen und Wählern die mit der Unionsbürgerschaft verknüpften Identitätsbezüge bewusst machen sollen.

Dies erinnert an den fundamentalen Zusammenhang zwischen kollektiver Identität und politischen Institutionen. ${ }^{51}$ Dass kollektive Identität sich institutionell und durch Repräsentanten objektivieren muss, sozusagen durch die Formierung eines kollektiven Surrogats für das der individuellen Identität entsprechende Ich, um nicht nur ein Wunsch oder eine Floskel zu bleiben, ist eine alte Sache. ${ }^{52}$ Umgekehrt brauchen Institutionen, auch und gerade politische, eine Verankerung in der bewussten Sinnwelt der Beteiligten und Betroffenen. Verfechter einer Konstitutionalisierung der Europäischen Union erhofften sich von dieser auch eine Festigung der europäischen Identität und der Chance der Identifizierung der Unionsbürger mit dem ,europäischen Projekt“ . ${ }^{53}$ Meistens dachten sie an den Abbau der ominösen „Entfremdung“ 54 eine Verfassung sollte das komplizierte Gebilde ,Europäische Union“ verständlicher und

49 So zum Beispiel Puntscher Riekmann/Wodak: „Europe for All“, 2003. Dies entspricht der „,identitätspolitischen“ Umsetzung der These, dass politische Identität allemal das jeweilige, momentane Produkt eines ständigen Diskussionsprozesses" ist. Vgl. Johannes Pollak: Zur politischen Identität der Europäischen Staatengemeinschaft, Frankfurt Main u.a. 1998. Das entspricht der These, dass sich auch auf europäischer Ebene „verfassungspatriotische Bindungen“ im „Medium der Politik selbst“ bilden und erneuern. Vgl. Jürgen Habermas: Ist die Herausbildung einer europäischen Identität nötig und ist sie möglich?, in: Jürgen Habermas (Hrsg.): Der gespaltene Westen. Kleine Politische Schriften X, Frankfurt Main 2004.

50 Thomas Meyer hält darüber hinaus auch ,emotionale Identifikationsangebote“ für nötig, die die Massen in ihren Bann ziehen und deren „Inszenierung“ nicht in den Prozessen politischer Interessenartikulation, Willensbildung, Entscheidung und Entscheidungsumsetzung aufgeht, vielleicht mit ihn gar nicht verknüpft sind. Im Blick auf beides legt sich die Frage nahe, ob das nicht auf die Produktion ,falschen Bewusstseins“ hinausläuft; innerhalb des Nationalstaats verhält es sich freilich nicht anders. Vgl. Meyer: Die Identität Europas, 2004.

51 Vgl. Hinweise auf die Dialektik von Institutionen und Sinnwelten bei Berger/Luckmann: Die gesellschaftliche Konstruktion der Wirklichkeit, 1969.

52 Vgl. die Hinweise bei Schneider: Europäische Identität, 1991.

53 Vgl. die Formulierung von Ulrich Haltern: Verfassungen speichern den Sinn einer (politischen) Gemeinschaft. Ulrich Haltern: Europäische Verfassung und europäische Identität, in: Ralf Elm (Hrsg.): Europäische Identität: Paradigmen und Methodenfragen, Baden-Baden 2002, S. 239-290, hier S. 255-256.

54 Siehe Zwischenbericht des Vorsitzenden der Reflexionsgruppe der Regierungskonferenz 1996 vom 24.08.1995. 
durchschaubarer machen, aber auch die demokratische Verantwortlichkeit der Organe und die normative Kraft der rechtlichen Grundordnung stärken, also den Charakter der Union als Rechtsgemeinschaft prägnanter vor Augen stellen. Außerdem sollte die Promotion dieser Grundordnung zur Verfassung auch die grundlegenden Sinngehalte den Bürgern ausdrücklicher und überzeugender nahe bringen. Dies ist namentlich die Funktion von Präambeln. Sie stellen sozusagen das Credo der in Verfassung gebrachten Gemeinschaft dar, machen grundlegende und wegweisende Identitätsgehalte des Gemeinwesens namhaft, die auch als Vorgaben und Rechtfertigungen für seine Politik fungieren. Dass dafür historische Erbschaften, gegenwartsbezogene Situationsbilder und Zukunftsperspektiven (erwartete Widerfahrnisse und den gemeinsamen Weg bahnende Projekte) infrage kommen, versteht sich von selbst.

Die Sache hat noch eine andere Seite. Verfassungen objektivieren nicht nur einigende Sinngehalte. Sie dokumentieren zwar prinzipielles Einvernehmen (als bestehend unterstelltes oder erstrebtes). Das muss nicht einen umfassenden (oder allen Dazugehörigen zugemuteten) Wertkonsens bedeuten; auch die Fixierung von Freiheitssphären geschieht von Verfassungswegen. Politische Systeme ohne gesicherten Respekt vor der Verschiedenheit sind totalitär. ${ }^{55}$ Zugleich regeln Verfassungen, wie man über das streitet, was keinen Konsens findet. ${ }^{56}$ Dass Streit integrieren kann, ist seit mehr als hundert Jahren selbstverständlich. ${ }^{57}$ Dies spielt mit, wenn die Politisierung der Europäischen Union, befürwortet wird, nämlich die Einbeziehung von grundsätzlichen und aktuellen Themen in die öffentliche Auseinandersetzung und die dadurch ausgelöste Aktivierung von Bürgern, gesellschaftlichen und politischen Kräften: Politisierung fördert Identität. In diesem Kontext steht auch das Thema der transnationalen politischen Öffentlichkeit als Voraussetzung für eine europäische , polity ${ }^{\text {. }}{ }^{8}$

Europäische Identität stimulierende Debatten können nicht nur konkrete integrationspolitische Vorhaben zum Gegenstand haben, sondern auch Auswahl und Akzentuierung der Komponenten einer die Identität definierenden europäischen Sinnwelt, namentlich die Kanonisierung des entsprechenden Credos. Das hat sich markant im Ringen um den Text des Verfassungsvertrags gezeigt - in solchen Vorgängen zeigt sich pointiert, dass nicht nur die normativen Prinzipien, die politischen Zielsetzungen und manche für ihre Realisierung maßgeblichen Parameter stets neu ausgehandelt werden müssen, sondern auch die Inhalte des „kulturellen Gedächtnisses“, soweit sie politisch sinngebende Relevanz haben können. ${ }^{59}$ Öf-

55 Vgl. zum Beispiel die Aussagen von Armin von Bogdandy und Robert Spaemann. Vgl. Bogdandy: Europäische und nationale Identität, 2003; Spaemann: Europa - Wertegemeinschaft oder Rechtsordnung?, 2001.

56 Siehe Stefan Korioth: Europäische und nationale Identität: Integration durch Verfassungsrecht, in: Veröffentlichungen der Vereinigung der Deutschen Staatsrechtslehrer, Band 62, Berlin 2003, S. 177-155, hier S. 127; dort in Fußnote 39 das Zitat von Helmut Dubiel, dem zufolge moderne demokratische Systeme „nicht auf Konsensus, sondern auf öffentlich inszeniertem Dissens" beruhen. Vgl. Helmut Dubiel: Unversöhnlichkeit und Demokratie, in: Wilhelm Heitmeyer (Hrsg.): Was hält die Gesellschaft zusammen? Bundesrepublik Deutschland: Auf dem Weg von der Konsens- zur Konfliktgesellschaft, Band 2, Frankfurt Main 1997, S. 425-446, hier S. 427.

57 Gleich zu Beginn seines klassischen Traktats über den Streit stellt Simmel fest: Der Kampf ist ,eigentlich die Abhülfsbewegung gegen den auseinanderführenden Dualismus, und ein Weg, um zu irgendeiner Art von Einheit zu gelangen“. Vgl. Georg Simmel: Soziologie, Leipzig 1908, S. 247. Zu der die Bürger in das politische Leben, das heißt in das Gemeinwesen, integrierenden Kraft des „Austrags politischer Kämpfe“, die die kollektive Identität des „miterlebenden Staatsvolks“ fördert. Vgl. zum Beispiel Rudolf Smend: Verfassung und Verfassungsrecht (1928), hier zitiert nach Rudolf Smend: Staatsrechtliche Abhandlungen und andere Aufsätze, 3. Auflage, Berlin 1994, S. 119-276, hier S. 151-157. Selbst „Querköpfe und Phantasten“ würden dabei als „Mitträger" des Gemeinwesens fungieren. Siehe ebenda, S. 157.

58 Bekanntlich betonten Gegner der Konstitutionalisierung der Europäischen Union im Zusammenhang mit der bekannten ,no demos ${ }^{6}$-These, mangels einer transnationalen politischen Öffentlichkeit sei eine demokratische Meinungs- und Willensbildung auf europäischer Ebene nicht möglich.

59 Siehe zum Beispiel Aleida Assmann: Nation, Gedächtnis, Identität - Europa als Erinnerungsgemeinschaft?, in: Simon Donig/Tobias Meyer/Christiane Winkler (Hrsg.): Europäische Identitäten - Eine europäische Identität?, Baden-Baden 2005, S. 24-32. 
ters hat man darauf hingewiesen, dass seinerzeit kaum ein Streitpunkt die Öffentlichkeit so sehr beschäftigt hat wie die Frage des Gottesbezugs oder der Bezugnahme auf das christliche (oder christlich-jüdische) Erbe Europas im Text der Präambel des Verfassungsvertrags. Es nimmt nicht wunder, dass nicht nur in den Konventsdebatten, sondern auch im damaligen literarischen Diskurs über die einigenden und wegweisenden Sinngehalte der europäischen Identität der Streit darüber engagiert ausgetragen wurde. ${ }^{60}$

Zur Sprache kamen in diesem Diskurs selbstverständlich auch viele andere Kandidaturen für die Aufnahme in das Mosaik identitätsrelevanter Gehalte: von der griechischen Philosophie - der klassischen und der stoischen - und der römischen Rechtskultur über Scheidung von weltlicher und geistlicher Autorität (im Mittelalter) und die Errungenschaften von Renaissance und Humanismus über die (leidvolle) Bewältigung der Glaubensspaltung, die Aufklärung, die Erbschaften der großen Revolutionen und die politischen Ideenkreise der späteren Neuzeit bis zu den Prinzipien der rechtsstaatlichen Demokratie und zum Leitbild der sozialen Marktwirtschaft.

Einige Fragen sind besonders beachtenswert:

- Gilt auch für das Europäertum ein anlässlich anderer kollektiver Identitätsbildungen immer wieder beobachtbarer Faktor, nämlich die profilierende Rolle von Antithesen, sozusagen Gegen-Identitäten (von den Perserkriegen für die alten Griechen über den Hundertjährigen Krieg für die Engländer und Franzosen bis zu heutigen Versuchen, Identitätsbildung auf Antithesen zu stützen)? ${ }^{61}$ Oder sei die Selbstverpflichtung Europas auf die Menschenwürde und die Menschenrechte (einschließlich der politischen und der sozialen) ein Beleg für den Verzicht auf Abgrenzung gegenüber außereuropäischen Ideen und ihren Ausgestaltungen? (Oder kommt in der Gleichsetzung europäischer und potenziell universaler Ordnungsprinzipien und Leitbilder ein expliziter oder zumindest impliziter expansiver ideeller Imperialismus zum Ausdruck?)

- Sollten nicht, neben Errungenschaften, auf die Europa stolz sein kann, weit mehr als üblich Erbschaften in das Identitätsbewusstsein aufgenommen werden, über die Europäer Scham empfinden müssten (die Verfolgung Andersdenkender, die Totalitarismen, Vertreibungen und Völkermorde), sozusagen als Herausforderungen an das europäische Gewissen $?^{62}$

Die zahlreichen konkurrierenden Hinweise auf historische Vermächtnisse dürfen freilich nicht davon ablenken, dass immer wieder auch darüber diskutiert wurde, in welchem Verhältnis vergangenheits-, gegenwarts- und zukunftsbezogene Komponenten der Konstruktion einer Schicksalsgemeinschaft zueinander stehen sollten.

60 Dass kirchliche Amtsträger sich für die Kanonisierung des christlichen Erbes aussprachen, ist ebenso selbstverständlich wie die Vertretung der Gegenposition durch Laizisten oder Verfechter der Ausblendung kultureller Bezüge der politischen Identität. Siehe zum Beispiel einerseits den damaligen vatikanischen ,Chefhistoriker" Walter Brandmüller und andererseits die Thesen von Enno Rudolph, dem Christentum einwohnenden theokratischen Tendenzen seien totalitär und die innerchristlichen Konflikte hätten sich zerstörerisch auf Europas Gemeinsamkeit ausgewirkt, oder die von Thomas Meyer aufgestellte These, im „Märchen“ von der christlichen Identität Europas sei „,kein Körnchen Wahrheit“ enthalten. Vgl. Walter Brandmüller: Integration Europas und Katholische Kirche, in: Ralf Elm (Hrsg.): Europäische Identität: Paradigmen und Methodenfragen, Baden-Baden 2002, S. 33-50; Enno Rudolph: Introduction, in: Furio Cerutti/Enno Rudolph (Hrsg.): A Soul for Europe. On the Cultural and Political Identity of the Europeans. An Essay Collection, Band 2, Leuven 2001, S. 1-12; Meyer: Die Identität Europas, 2004.

61 Man kann sich an den Versuch von Habermas und anderen erinnern, die Absetzung von den USA in diesem Sinn zu deuten.

62 Siehe zum Beispiel die pointierte Hervorhebung des Holocaust bei Schmale: Geschichte und Zukunft der Europäischen Identität, 2008. 


\section{Folgerungen für heute und morgen}

Das Diskursfeld der vergangenen Dezennien ist bunt und vielfältig. Was davon zu halten ist, klärt sich womöglich leichter, wenn man wahrnimmt, wer sich engagiert hat und warum. Einfach liegt der Fall bei den Befürwortern einer politischen Verdichtung und Vertiefung der Union, seien sie in der Politik oder im akademischen Leben verankert. ${ }^{63}$ Ebenfalls klar ist die Bewandtnis, wenn sich Autoren (und Akteure) für eine bestimmte inhaltlich-ideelle Ausrichtung der Europäischen Union engagieren, zum Beispiel für ein „,christliches Europa“, und dieses Anliegen in das Bewusstsein der Unionsbürgerinnen und -bürger oder in das offizielle Credo einbringen wollen, was nicht geradewegs auf missionarischen Absichten beruhen muss, sondern unterschiedlich motiviert sein kann. ${ }^{64}$ Entsprechende Plädoyers rufen unter Umständen alsbald Gegner auf den Plan, die für eine säkular-liberale ideelle Ausrichtung der Union plädieren. Etwas anders verhält es sich mit Plädoyers für eine Kräftigung der europäischen Identität, die auf eine Kritik der technokratischen und marktliberalen Prägung der Europapolitik ausgehen. ${ }^{65}$

Diese und noch manche andere Intentionen und Darlegungen machen kaum den Eindruck, als würde die jetzt aktuelle Europapolitik auf sie warten.

Eine recht radikale Antithese zu diesem komplexen ,main stream'sollte aus dem Rückblick nicht ausgeblendet werden, sie stammt namentlich von Ulrich Haltern. Die üblichen Maßnahmen der Identitätspolitik hätten, so Ulrich Haltern, ,etwas Unbeholfenes“ und ,entbehrten nicht eine gewissen Komik“; als Versuche, die neureiche „Gesichts- und Geschichtslosigkeit der Union“ zu überwinden. Nicht von ungefähr seien Identitäten in der heutigen Welt unverbindlich geworden, hätten Warencharakter gewonnen. Das Einkaufen, Konsumieren und Reisen seien die wichtigsten sozialen Aktivitäten. Die Funktion der Europäischen Union sei es, diese zu ermöglichen, nicht mehr, nicht weniger. Ihre Rationalität habe mit dem ,politischen Sinn in der klassisch imaginierten Form nichts zu tun".66 Der Autor meint am Ende: „Wohin dies führen mag, kann man nicht vorhersehen“; ein „Umschwung" in dem das Politische im alten Sinn wiedererstehen könnte, ${ }^{67}$ sei möglich, aber

63 Selbstverständlich kann die Fortbildung der Union zu einer , polity“ im Dienst von vielerlei Anliegen gewünscht werden - von der Stärkung der Handlungsfähigkeit nach außen bis zur Wiederherstellung des ,Primats der Politik“ gegenüber der Dynamik transnationaler Marktkräfte. So zum Beispiel des Öfteren Jürgen Habermas; siehe zum Beispiel schon folgende Beiträge: Jürgen Habermas: Die postnationale Konstellation und die Zukunft der Demokratie, in: Blätter für deutsche und internationale Politik 7/1998, S. 804-817; Jürgen Habermas: Der europäische Nationalstaat unter dem Druck der Globalisierung, in: Blätter für deutsche und internationale Politik 4/1999, S. 425-436.

64 Beispielsweise um die Bevölkerungen christlich geprägter Länder zu gewinnen, wie Irland und Polen, oder auch um mithilfe der Exklusion nichtchristlicher Länder die Aufnahmechancen der Türkei zu mindern.

65 Ein derartiger, Euro-Kommunitarismus` ist nicht mit dem althergebrachten oder mit einem erneuerten ,EuroFöderalismus' gleichzusetzen.

66 Laut Ulrich Haltern werden Identitäten ,designt, anprobiert, einen Abend lang getragen und dann gegen andere getauscht“, sie seien ,notwendig flüchtig“, - es wäre vielleicht gut, wenn die Europäische Union ,,den scheiternden und unglaubwürdigen ,Europa der Bürger'-Diskurs endlich aufgäbe“. Sie könnte das erste Gemeinwesen sein, das sich an die Bedingungen der heutigen Existenz seiner Bürger anpasst“ und ,auf Erzählungen von geteilten Werten und historisch verwurzelter Gemeinschaftlichkeit“ verzichtet. Es gehe nicht darum, „,eine Identität zu konstruieren“, sondern ,die Festlegung zu vermeiden und sich die Optionen offen zu halten“. Vgl. Haltern: Verfassungen speichern den Sinn einer (politischen) Gemeinschaft, 2002, S. 268, 270, 274, 280-288.

67 Der Autor verweist auf die Topoi des Eros, der ,Investition des Körpers“ und des Opfers hin zum Einsatz der Hingabe des Lebens. Haltern: Verfassungen speichern den Sinn einer (politischen) Gemeinschaft, 2002, S. 287-288. Anderwärts hat er seine Sicht inzwischen bekräftigt: Das ,postmoderne Subjekt“ sei „ohne eine gesicherte, wesentliche oder anhaltende Identität konzipiert“. Mit der kollektiven Identität verhalte es sich ähnlich. Auch beim Staat verliere ,,das Politische sein Geheimnis, seinen Sog und seine Neigung zu Hypertrophie und Gewalt, auf der EU-Ebene habe es dies nie gegeben. Nun sei man sich des Vertrauens, ,,atavistische Politikmuster überwunden zu haben“, nicht mehr sicher. Massive Akzeptanzverweigerungen und Unruhen in etlichen Mitgliedstaaten, die eine europäische Dimension aufwiesen, hätten die Grenzen der Integration gezeigt - 
das würde dann wohl die Stunde des Staates sein. Im Unterschied zu der oben angesprochenen, die transnationale Identitätsstärkung fördernden Politisierung der Europapolitik, die in Halterns Perspektive als eine fragwürdig ästhetisierende Inszenierung erscheint, würde eine solche Politisierung die Union wohl in eine Existenzkrise stürzen.

Das ist hier vielleicht deshalb von Belang, weil die im vorletzten Abschnitt skizzierten Entwicklungen der Europapolitik dadurch in ein neues Licht rücken.

\section{Die Zukunft der Europäischen Union: erzwungene Schicksalseinheit oder bejahte Solidarität?}

Die Integration ist in einen bemerkenswerten Ernstfall geraten: ${ }^{68}$ Die Unterstellung eines Mitgliedstaates ,unter Zwangsverwaltung " ${ }^{69}$ gehört sicher nicht in den Bereich der politischen Normalität. Manche 2010 getroffene Entscheidungen der Staats- und Regierungschefs werden als Akte des Vertragsbruchs betrachtet. ${ }^{70}$ Zwei Parolen wurden ausgegeben: Erstens: Nicht nur die gemeinsame Währung stehe auf dem Spiel, sondern die Union. Zweitens: Es gebe keine Alternative. In den Augen der Staats- und Regierungschefs war ein Ausnahmezustand gegeben, in dem man über die für den Normalzustand geltenden Rechtsnormen hinweggehen muss. Dafür gibt es zwei Möglichkeiten: erstens die kommissarische Ermächtigung (oder Machtergreifung) für den Notfall mit der Maßgabe der unverzüglichen Rückkehr zum normativen status quo ante, wenn die existenzielle Gefahr gebannt ist; zweitens die definitive Inanspruchnahme der Souveränität. ${ }^{71}$ Einer bekannten Lehre zufolge hängt die Konstituierung des neuzeitlichen souveränen Staates mit dem Erfordernis der Bewältigung ,,bedrohlicher Lagen“ zusammen, die zur Emanzipation der Abkoppelung der Staatsgewalt von überkommenen normativen Bindungen führte. ${ }^{72}$ Kriterien dafür waren erstens die Notwendigkeit, zweitens die Außerordentlichkeit. Die mit der Notwendigkeit konfrontierte souveräne Staatsgewalt war im Ursprung außerordentliche Gewalt. ${ }^{73}$

Mit Blick darauf, dass die Staats- und Regierungschefs im Jahre 2010 eine dem in mancher Hinsicht entsprechende Entscheidungshoheit kollektiv in Anspruch genommen haben, ist zweierlei bedeutsam: Erstens setzten sie sich, wie gesagt, von einer außerordentlichen Notwendigkeit ausgehend, über Bestimmungen des Unionsrechts hinweg, sie machten also

die Wiederkehr der Gewalt sei nicht ausgeschlossen. Gleichwohl sei „die Möglichkeit eines genuin politischen Europa nicht ausgeschlossen“, entweder indem ,ursprünglich staatliche Imaginationen auf die supranationale Ebene“ verschoben werden oder auch ,im Nationalstaat eine postsouveräne Imagination losgelöst vom Souveränitätsdenken ermöglich[t]“" werden; ,,schließlich könnte es zu hybriden Konstellationen kommen.“ Ulrich Haltern: Finalität, in: Armin von Bogdandy/Jürgen Bast (Hrsg.): Europäisches Verfassungsrecht. Theoretische und dogmatische Grundzüge, 2. Auflage, Dordrecht/Heidelberg/London/New York 2009, S. 279-334, hier S. 327-331.

68 Siehe oben die Abschnitte „Die jüngste Krise, ihre Bewältigung und das Erfordernis von Solidität und Solidarität“ und „Wird die Sanierungsrechnung ohne den Bürger gemacht?“.

69 Dieser Ausdruck wurde in der Medienberichterstattung geläufig. Siehe zum Beispiel Cerstin Gammelin: EU stellt Griechenland unter Zwangsverwaltung - Um den Euro zu schützen, greift Brüssel zum ersten Mal in die Haushaltshoheit eines Mitgliedstaates ein, in: Süddeutsche Zeitung, 04.02.2010.

70 Gegen Jahresende 2010 hat die französische Finanzministerin Christine Lagarde offen einbekannt, dass sowohl die Griechenlandhilfe wie der „Rettungsschirm“ vertraglich nicht gedeckt waren. Vgl. Süddeutsche Zeitung: „Wir werden bedingungslos sparen“, Interview mit Christine Lagarde, 23.12.2010; Thiemo Jeck/Bert van Roosebeke/Jan S. Vosswinkel: Keinen Euro nach Athen tragen, Centrum für Europäische Politik: cepStudie, März 2010; Martin Seidel: Aktuelle Probleme der europäischen Währungsunion, in: integration 4/2010, S. 334-349, vor allem S. 344-348.

71 Siehe Peter Schneider: Ausnahmezustand und Norm - Eine Studie zur Rechtslehre von Carl Schmitt, Stuttgart 1957, S. 110.

72 Siehe statt vieler Herbert Krüger: Allgemeine Staatslehre, Stuttgart 1964, S. V-VII

73 Ebenda, S. 25-29. Krüger vermerkt, dass noch John Locke einer solchen Gewalt den Anspruch auf Gehorsam abspricht - sie gehöre in den Naturzustand. 
von ihrer Position als ,Herren der Verträge“ auf eine ungewöhnliche Weise Gebrauch. ${ }^{74}$ Zweitens haben sie es fertig gebracht, sich - im Anschluss an die Sofortmaßnahmen zur Bewältigung der unmittelbar bedrohlichen Lage - über politische Differenzen hinwegzusetzen, die bisher als kaum überwindbar galten. Was die Annäherung von Politiken betrifft, agierte das deutsch-französische Tandem avantgardistisch. Sollten sich die Absichten durchsetzen lassen, dann könnte man den Eindruck einer Fusion gewinnen. Noch ist nicht absehbar, ob und wie die koordinierte Steuerung der Wirtschaftspolitiken so ausgestaltet wird, dass sie als eine ,Politik aus einem Guss“ erscheint. Das gibt der von Wolfgang Wessels entwickelten These vom „fusionierten Föderalstaat“ eine neuartige Relevanz. ${ }^{75}$ Der sich dabei darbietende Komplex von Staatsgewaltausübung schillert gewissermaßen zwischen Singular und Plural, zwischen dem „fusionierter Mehrebenenstaat“ und Plural (die Staaten betreiben ein System, das sie als Strategie für konkrete Problemlösungen arrangiert und konsolidiert haben). Die Instrumente ,erscheinen wie von nur einem Akteur eingesetzt ${ }^{“} .{ }^{76}$ Das bedeutet einiges mit Blick auf unser Thema.

Erstens hat die aktuelle europapolitische Entwicklung die Antwort auf die Frage, in deren Kontext die Identitätsdiskussion stattfand, in einen neuen Bedingungszusammenhang gestellt, nämlich die Frage nach dem faktischen und dem wünschenswerten Charakter der Europäischen Union.

Das politische System der Europäischen Union stellt sich als ein Mehrebenengebilde dar, das eindeutiger als bisher dem Grundkonzept des bloßen Not- und Verstandesstaats entspricht. Emphatischere Rechtfertigungen, wie sie von Identitätskonstruktionen geleistet werden sollen, treten in den Hintergrund. Die auf unvermeidlicher Notwendigkeit beruhende Schicksalsgemeinschaft (das ,Sitzen im gleichen Boot") hat mehr Gewicht als andere gemeinsame Betroffenheit und damit identitätstiftende Merkmale der Gegenwartslage, von identitätsstärkenden ideellen und politischen Vermächtnissen aus der Vergangenheit gar nicht zu reden.

Zweitens: Die engere horizontal und vertikal verzahnte Ausübung von Hoheitsgewalt macht es den Bürgerinnen und Bürgern schwer, sich ein Bild von Verantwortlichkeiten zu machen, konkrete Verantwortungsträger für wichtige Entscheidungen zu identifizieren und, wenn schon, zwischen nationalen und europäischen Amtskompetenzen zu unterscheiden. Das kann die, Eurokratie" noch intransparenter erscheinen lassen. Dies kann - trotz medialer Präsentation von Per-

74 Zuvor handelte es sich bei dieser Qualifizierung um eine Formel, mir der vor allem die Behauptung von Gegnern der Unionsverträge abgewiesen werden sollte, die Reformen brächten die Umwandlung des „Staatenverbundes" in einen Bundesstaat, der den Mitgliedstaaten die Souveränität nähme. Wenn in Verbindung damit betont wurde, die Staaten könnten die Union, wenn sie das wollten, einvernehmlich auflösen, hielt kaum jemand das für eine ernsthaft infrage kommende Möglichkeit.

75 Sie besagt unter anderem: Die Staatsgewalt der Mitgliedstaaten der Europäischen Union wird, unter Mitwirkung insbesondere verschiedener nationaler, supranationaler und unter Umständen auch anderer Organe und Akteure so ausgeübt, dass man von ,,shared governance“ sprechen kann; sie wachsen ,entsprechend den Notwendigkeiten übergreifender Problemverarbeitung zusammen“, wobei der Kommunikations- und Interaktionszusammenhang durchaus von Spannungen durchzogen ist (was sich in vielfältigem Tauziehen geltend machen kann). Vgl. Heinrich Schneider: Die Fusionsthese: Voraussetzungen, Eigentümlichkeiten, Implikationen - Ansätze zur Analyse des integrationstheoretischen Zentralbegriffs von Wolfgang Wessels, in: Udo Diedrichs/ Anne Faber/Funda Tekin/Gaby Umbach (Hrsg.): Europe Reloaded. Differentiation or Fusion?, Baden-Baden 2011, S. 23-77.

76 Wolfgang Wessels Rede von Erscheinungsweisen der Praktizierung fusionierter Hoheitsgewalt(-en) korrespondiert bemerkenswert mit der Darlegung Ulrich Halterns über die nationalstaatliche, die supranationale und die „,hybride“ Politik-,,Imagination“. Die Unklarheit, ob ein Akteur (im Singular) handelt oder ob der Betrachter einen Handlungsverbund mehrerer Akteure vor sich hat, schrieb übrigens Georg Jellinek schon dem Staatenbund zu. Vgl. Haltern: Finalität, 2009, S. 327-331; Georg Jellinek: Allgemeine Staatslehre, 6. Neudruck der 3. Auflage, Darmstadt 1959. 
sönlichkeiten wie unter anderem Angela Merkel und Nicolas Sarkozy - zur Stärkung der vielberufenen Entfremdung führen und die Legitimität des EU-Systems noch mehr infrage stellen. ${ }^{77}$

Drittens: Dies gilt erst recht, wenn unklar ist, ob die Inanspruchnahme der außerordentlichen Notstandskompetenz wirklich ein Ausnahmevorgang bleibt oder zur Etablierung einer kompakten Kollektivsouveränität der ,Herren des Systems' führt, mit der Folge der Schwächung der normativen Kraft des Unionsrechts. Die Folge wäre ein weiterer Legitimationsverlust; gerade weil die Europäische Union nicht im gängigen Sinn demokratische Legitimität besitzt, ist die rechtsnormative für sie umso wichtiger.

Viertens: Die angesichts der jüngsten Krisen ausgerufene Schicksalsverbundenheit bringt weder eine Konvergenz der Einstellungen zustande, noch beruht sie auf einer solchen. Sie bedeutet für die einen eine Rettungschance, allerdings verknüpft mit erheblichen Unannehmlichkeiten, für die anderen ein Verhängnis - jeweils nach Maßgabe der materiellen Interessen. Das bewirkt aber eine gegensätzliche (antagonistische) Einschätzung des ,Sitzens in einem Boot`

Das alles brächte die Wiederaufnahme der Identitätsdebatte in einen veränderten Kontext, womöglich unter dem Motto ,Zuerst kommt das Überleben und dann die Moral'. Man könnte sich an eine aus der Frühzeit der Sozialpsychologie stammende Einsicht erinnern, die sich auf die Faktoren kollektiver Identitätsbildung bezieht: ${ }^{78}$ Nichts ist so wirksam wie das Bewusstsein, einem gemeinsamen Feind gegenüberzustehen; ${ }^{79}$ etwas weniger, aber immer noch recht motivkräftig ist das Bewusstsein gemeinsamer Not. Deutlich geringere Bindekräfte aktiviert das Wissen um gemeinsame Interessen (also der Gemeinsamkeit von Vorteilen und Nachteilen, die zwar als wichtig, jedoch nicht als überlebenswichtig wahrgenommen werden). Erst am unteren Ende der Skala des Motivationsgewichts vereinigender Kräfte liegt die Gemeinsamkeit von Werten und Ideen.

War also die Identitätsdebatte des vergangenen Jahrzehnts eine Sache naiver Idealisten oder klug agierender Mythologieproduzenten, deren Konstrukte nun durch die harte Realität als fragwürdig erwiesen werden? Nötigt diese Realität zu einer eher nüchternen, desillusionierten Wahrnehmung des Identitätsproblems?

Folgerungen für unser Problem sollten nicht vorschnell abgeleitet werden. Zum einen ist es nicht ausgemacht, dass die Befunde der Gruppenpsychologie ohne Weiteres auch auf der transnationalen Makro-Ebene gelten. Schon im politischen Gemeinwesen ist Solidarität nicht nur interpersonal und in ,face-to-face'-Beziehungen wirksam, sondern wird normativ stabilisiert. ${ }^{80}$ Zum anderen hat es mit Auswirkung der gemeinsamen Not auf die Kohäsion

77 Wolfgang Wessels hält die Legitimitätsproblematik des Fusionssystems für wichtig; er präsentiert zwei alternative Sichtweisen: „Erosion“ und „Akzeptanzsicherung durch Partizipation“. „Erosion“ ist durch mangelnde „Transparenz der Problemverarbeitungsprozesse“, durch fehlende „Eindeutigkeit der Verantwortungszuordnung “ gekennzeichnet; es gebe keine „Identifikationsmöglichkeit der Bürger“. Die „Akzeptanzsicherung durch Partizipation“ bezieht sich wohl eher auf den normalen Willensbildungs- und Umsetzungsprozess, in den ja - für das Fusionssystem typisch unterschiedliche Akteure auf mehreren Ebenen eingebunden sind. Im Ausnahmezustand ist das anders.

78 Vgl. Schneider: Europäische Identität, 1991. Im Blick stehen dabei die experimentell erhobenen Befunde von Muzafer Sherif über die Faktoren effektiver Gruppenkohäsion.

79 Dem entspricht auf der Makro-Ebene der Befund der Historiker, dass nationale Identitäten sich immer wieder gegenüber einer Feindnation konstituiert haben. So zum Beispiel Orest Ranum: Counter-Identitities of Western European Nations in the Early-Modern Period: Defintions and Points of Departure, in: Peter Boerner (Hrsg.): Concepts of National Identity, Baden-Baden 1986, S. 63-78. Die Folgerung liegt nahe: Ein probates Mittel zur Identitätsstärkung ist es, Feindbilder zu nutzen, gegebenenfalls zu konstruieren, eventuell auch ,innere‘.

80 Das wussten schon die antiken politischen Theoretiker: Bei Aristoteles unterscheidet sich die ,philía politiké von der unmittelbar zwischenmenschlichen ,philía“ dadurch, dass sie, modern gesprochen, auf einer Gemeinsamkeit der Sinnwelt (,homónoia') beruht. Sie bewirkt Interessengemeinsamkeit (Einvernehmen über das „sýmpheron“, und normative Übereinstimmung (nämlich über das ,díkaion’ und das ,ádikon'). Demgemäß definiert Cicero den „,populus“ als durch den „,consensus iuris“ und durch die „,communio utilitatis“ geeint. Der griechischen „homónoia“ entspricht die römische „concordia“. 
eine besondere Bewandtnis, wenn diese Not nicht auf einem unvorgreiflichen Schicksal beruht, sondern entweder Folge eines Fehlverhaltens von Teilkollektiven des Gemeinwesens oder als Konsequenz einer als verhängnisvoll eingeschätzten Politik betrachtet wird. ${ }^{81}$ Allemal stärkt ein bejahtes Wir-Bewusstsein den Zusammenhalt einer Gruppe oder eines Gemeinwesens und die Realisierungschance von Solidaritätszumutungen. Ganz abgetan ist die Frage nach der kollektiven Identität der Europäerinnen und Europäer jedenfalls nicht.

81 Im Extremfall können dann die für diese Politik Verantwortlichen als Sündenböcke, das heißt als Feindsurrogate, angesehen werden. 